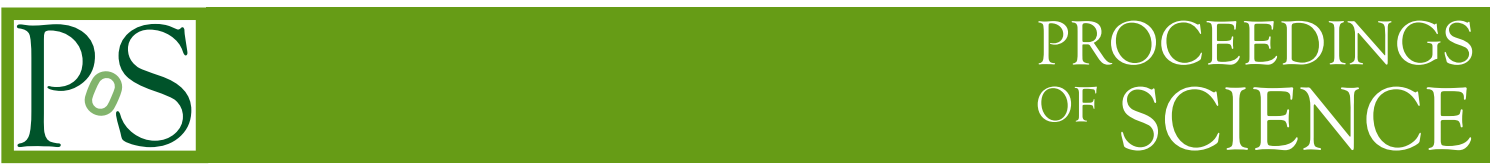

\title{
Searches for long-lived particles in ATLAS
}

\section{Masahiko Saito ${ }^{a, *}$ on behalf of the ATLAS Collaboration}

${ }^{a}$ International Center for Elementary Particle Physics (ICEPP), The University of Tokyo, 7-3-1 Hongo, Bunkyo-ku, Tokyo 113-0033, Japan

E-mail: masahiko.saito@cern.ch

Several models beyond the Standard Model predict new particles which could have a significant lifetime owing to the mass-degeneracy, heavy intermediate particles or small coupling to the lighter particles. When such a long-lived particle decays far from a beam collision point, it is hard for standard event reconstruction targeting promptly decaying signals to detect it, in spite of the fact that a long-lived particle would leave a characteristic signature in the detector. To extend the sensitivity for such various BSM models, the ATLAS collaboration performs searches for long-lived particles using dedicated techniques to reconstruct the characteristic signatures. This paper reviews recently published results of searches for long-lived particles with displaced signatures using data collected by the ATLAS detector in 2015 and 2016 at the LHC.

The Eighth Annual Conference on Large Hadron Collider Physics-LHCP2020

25-30 May, 2020

online

\footnotetext{
${ }^{*}$ Speaker
} 


\section{Introduction}

Experiments at the energy frontier have been searching for new physics and new particles beyond the Standard Model (BSM). No signs of BSM, however, has been found. The importance of searches using unconventional signatures increases to cover as large phase spaces as possible. Searching for long-lived particles is one of the typical examples.

Long-lived particles appear in many BSM models, owing to the mass-degeneracy with the lighter particles, the heavy intermediate virtual particles, or small couplings to the SM sector. Depending on their lifetime, decay products, and quantum charges, the long-lived particles can be observed as various characteristic signatures. Since the standard event reconstruction of ATLAS [1] is not designed to find such signatures, reconstruction of long-lived particles requires dedicated techniques, including trigger, reconstruction, identification and background estimation for non standard physics objects.

This paper reviews two searches for long-lived particles recently published by the ATLAS collaboration using $36.1 \mathrm{fb}^{-1}$ of $p p$ collision data at the LHC. Both analyses target long-lived neutral BSM particles decaying within the ATLAS detector and observed as displaced signatures.

\section{Search for light long-lived neutral particles decaying into collimated leptons or light hadrons}

This analysis [2] uses $36.1 \mathrm{fb}^{-1}$ of $p p$ collision data collected in 2015 and 2016, and targets light long-lived neutral particles decaying into groups of fermions. Such a particle is known as a dark photon which is weakly mixing to the SM photon. Due to the small couplings to the SM sector, a dark photon becomes a long-lived particle, and its lifetime varies depending on the coupling strength. Assuming a dark photon is light, it is highly boosted, and results in collimated groups of decay products, called Dark Photon Jet (DPJ). Depending on the decay position in the detector, different types of DPJ signatures will be observed. This analysis focuses on DPJs decaying after the electromagnetic calorimeter, and categorizes DPJs into two types: DPJ reconstructed using calorimeter energy deposits ( $h \mathrm{DPJ})$ and DPJ reconstructed using muon spectrometer tracks $(\mu \mathrm{DPJ})$.

$h \mathrm{DPJ}$, targeting dark photons decaying into electron or pion pair in the hadron calorimeter, is identified using characteristic jets with low electromagnetic fraction, defined as the ratio of the energy deposited in the electromagnetic calorimeter to the total jet energy. The requirement of low electromagnetic fraction ensures the jet comes from the dark photon decays in the hadronic calorimeter. The events having a $h$ DPJ can be triggered by a "Calorimeter Ratio trigger" [3] which requires high $p_{\mathrm{T}}$ single jets $\left(p_{\mathrm{T}}>60 \mathrm{GeV}\right.$ ) having large hadron/electromagnetic calorimeter energy ratio with no inner detector tracks within $\Delta R<0.2$.

$\mu \mathrm{DPJ}$, mainly targeting dark photons decaying into muons, is reconstructed from at least two tracks reconstructed in the muon spectrometer. The events having a $\mu$ DPJ can be triggered by a trimuon MS-only trigger [3], requiring at least three tracks reconstructed in the muon spectrometer, and a muon narrow scan trigger, requiring a single high $p_{\mathrm{T}}(>20 \mathrm{GeV})$ muon at the first level hardware-based trigger (L1), then searching for the 2nd muon $\left(p_{\mathrm{T}}>6-15 \mathrm{GeV}\right)$ in a cone of $\Delta R=$ 0.5 around the L1 muon at the following high level software-based trigger (HLT). 
This analysis searches for the process of $H \rightarrow 2 \gamma_{d}+X$ and $H \rightarrow 4 \gamma_{d}+X$, where $H$ is either of the SM Higgs boson or a BSM heavy scalar boson. Since two dark photons or two collimated dark photon pairs are pair-produced, three signal regions are defined by a pair of DPJ types: $\mu$ DPJ- $\mu$ DPJ, $\mu \mathrm{DPJ}-h \mathrm{DPJ}$ and $h \mathrm{DPJ}-h \mathrm{DPJ}$. Signal efficiencies at the optimal dark photon lifetime for each region vary from $10^{-4}$ to $10^{-1}$ depending on the mass of $H\left(m_{H}\right)$ and decay process $\left(2 \gamma_{d}\right.$ or $\left.4 \gamma_{d}\right)$ mainly because of trigger requirements. The main background sources are cosmic muons for $\mu \mathrm{DPJ}$ and multi-jets events for $h \mathrm{DPJ}$, and they are rejected using a boosted decision tree (BDT) classifier. The yield of the remaining backgrounds is estimated by using control regions. The expected number of background events in the signal regions are $128 \pm 26$ (stat.) \pm 3 (syst.) $(\mu \mathrm{DPJ}-\mu \mathrm{DPJ}), 177 \pm$ 86 (stat.) \pm 4 (syst.) $(\mu \mathrm{DPJ}-h \mathrm{DPJ})$, and $97 \pm 48$ (stat.) \pm 2 (syst.) $(h \mathrm{DPJ}-h \mathrm{DPJ})$, and the observed numbers are 133, 179, and 69, respectively. No significant excess was found in all signal regions.

95\% CL upper limits were set on $\sigma \times \mathrm{B}$ as a function of the dark photon proper decay length as shown in Figure 1 (a). Figure 1 (b) shows the exclusion regions as a function of the dark photon mass and of the kinetic mixing parameter with the SM photon $(\epsilon)$. This analysis excludes a lower $\epsilon$ region, corresponding to the longer lifetime phase space, which was not covered by Run 1 analyses: prompt lepton-jet analysis [4] and displaced lepton-jet analysis [5].

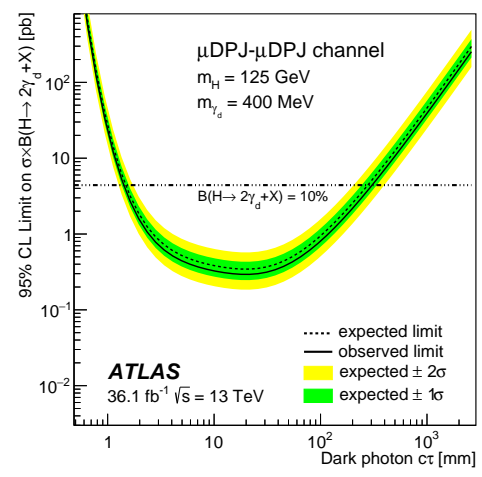

(a)

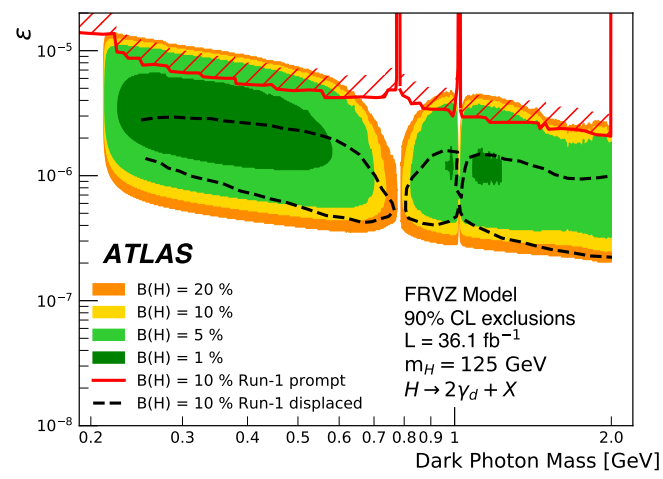

(b)

Figure 1: (a) Upper limits at 95\% CL on the cross section times branching fraction for the process $H \rightarrow 2 \gamma_{d}+X$ in the $\mu$ DPJ- $\mu$ DPJ final states for $m_{H}=125 \mathrm{GeV}$. (b) $90 \% \mathrm{CL}$ exclusion regions for the decay $H \rightarrow 2 \gamma_{d}+X$ as a function of the $\gamma_{d}$ mass and of the kinetic mixing parameter $(\epsilon)$, assuming the FRVZ model [6,7] with decay branching fractions of $H \rightarrow 2 \gamma_{d}$ between $1 \%$ and $20 \%$.

\section{Search for long-lived neutral particles decaying into displaced hadronic jets in the ATLAS inner detector and muon spectrometer}

This analysis [8] uses $33 \mathrm{fb}^{-1}$ of $p p$ collision data collected in 2016, and targets a process where a pair of long-lived neutral scalar particles $(s)$ decay into SM fermion pairs. Such a long-lived scalar particle appears in several BSM scenarios predicting a hidden sector (HS). Depending on the effective Yukawa coupling between the $s$-boson and the SM particles, the $s$-boson may have a significant lifetime. This analysis focuses on the case where one of the long-lived scalars decays in the inner detector and another one decays in the muon spectrometer. The former decay is tagged by 
a displaced vertex in the inner detector (IDVx) and the latter one is tagged by a displaced vertex in the muon spectrometer (MSVx).

Events are triggered by a dedicated muon trigger ("Muon RoI Cluster trigger") [3], which requires two muons $\left(p_{\mathrm{T}}>10 \mathrm{GeV}\right)$ at $\mathrm{L} 1$, and then requires three (four) muons within $\Delta R=0.4$ in the barrel (endcap) of the muon spectrometer at HLT. Then, at an offline reconstruction step, dedicated displaced vertex reconstruction algorithms are used for both IDVx and MSVx. IDVx is reconstructed from tracks including large radius tracks [9, 10]. MSVx is reconstructed using at least three muon spectrometer tracklets [11]. Trigger efficiencies range from $2 \%$ to $16 \%$, and the overall efficiencies range from $0.003 \%$ to $0.09 \%$ for several typical signal mass points.

The background yield in the signal region is estimated by applying the probability that background events contain good IDVx into the control region which is defined as the same as the signal region except for the requirement of the presence of an IDVx. The observed probability is $(7.4 \pm$ 1.1 (stat.) $) \times 10^{-6}$, and the expected number of events in the signal region is $1.16 \pm 0.17$ (stat.) \pm 0.29 (syst.). One event is observed, and it is consistent with the estimated background yield.

Figure 2 (a) shows the upper limits for $B_{H \rightarrow s s}$ as a function of the new scalar particle proper lifetime (c $\tau$ ). Figure 2 (b) is a comparison plot with the previous two searches, MS analysis [12] and Calorimeter Ratio analysis [13]. This analysis increases sensitivity to low-mass scalars with shorter proper lifetimes.

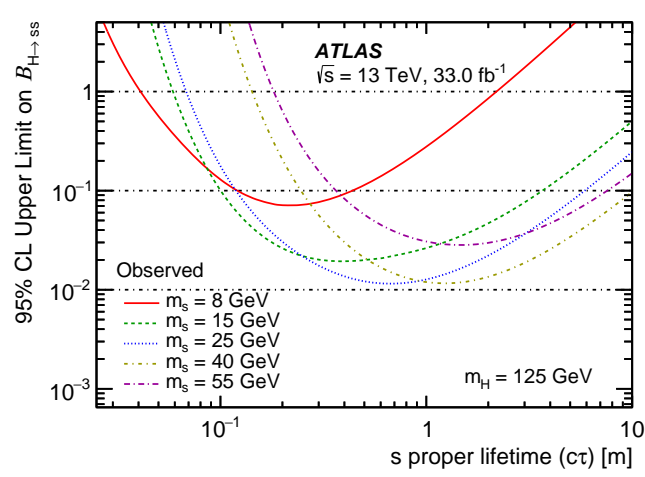

(a)

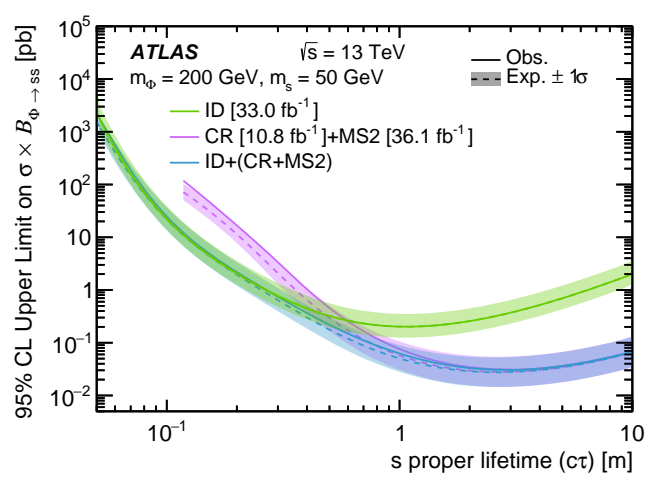

(b)

Figure 2: (a) Upper limits at $95 \% \mathrm{CL}$ on the branching ratio $\left(B_{H \rightarrow s s}\right)$ for $m_{H}=125 \mathrm{GeV}$ with several long-lived neutral scalar masses $\left(m_{S}\right)$, assuming the SM Higgs boson gluon-gluon fusion production cross section of $49 \mathrm{pb}$. (b) Combined limits from this analysis (ID) and the CR [13] and MS [12] analyses for $m_{\Phi}$ $=200 \mathrm{GeV}$ decaying into $50 \mathrm{GeV}$ scalars.

\section{Summary}

Searches for long-lived particles are being performed to extend the sensitivities for new physics beyond the Standard Model, by using several dedicated techniques to identify BSM particles. This paper reviews two recently published results using data collected in 2015 and 2016 with the ATLAS detector, and no deviations from the SM are reported. The ATLAS collaboration continues to develop new techniques and to analyze full Run 2 data for various signal signatures, including ones not shown in this paper, to extend the discovery reach. 


\section{References}

[1] ATLAS Collaboration, The ATLAS Experiment at the CERN Large Hadron Collider, JINST 3 (2008) S08003.

[2] ATLAS Collaboration, Search for light long-lived neutral particles produced in $p p$ collisions at $\sqrt{s}=13 \mathrm{TeV}$ and decaying into collimated leptons or light hadrons with the ATLAS detector, Eur. Phys. J. C 80 (2020) 450, arXiv: 1909.01246 [hep-ex].

[3] ATLAS Collaboration, Triggers for displaced decays of long-lived neutral particles in the ATLAS detector, JINST 8 (2013) P07015, arXiv: 1305.2284 [hep-ex].

[4] ATLAS Collaboration, A search for prompt lepton-jets in pp collisions at $\sqrt{s}=8 \mathrm{TeV}$ with the ATLAS detector, JHEP 02 (2016) 062, arXiv: 1511.05542 [hep-ex].

[5] ATLAS Collaboration, Search for long-lived neutral particles decaying into lepton jets in proton-proton collisions at $\sqrt{s}=8 \mathrm{TeV}$ with the ATLAS detector, JHEP 11 (2014) 088, arXiv: 1409.0746 [hep-ex].

[6] A. Falkowski, J. T. Ruderman, T. Volansky, and J. Zupan, Hidden Higgs decaying to lepton jets, JHEP 05 (2010) 077.

[7] A. Falkowski, J. T. Ruderman, T. Volansky, and J. Zupan, Discovering Higgs Boson Decays to Lepton Jets at Hadron Colliders, Phys. Rev. Lett. 105 (2010) 241801.

[8] ATLAS Collaboration, Search for long-lived neutral particles produced in pp collisions at $\sqrt{s}=13 \mathrm{TeV}$ decaying into displaced hadronic jets in the ATLAS inner detector and muon spectrometer, Phys. Rev. D 101 (2020) 052013, arXiv: 1911.12575 [hep-ex].

[9] ATLAS Collaboration, Performance of the reconstruction of large impact parameter tracks in the inner detector of ATLAS, ATL-PHYS-PUB-2017-014, 2017, https://cds. cern.ch/record/2275635.

[10] ATLAS Collaboration, Performance of vertex reconstruction algorithms for detection of new long-lived particle decays within the ATLAS inner detector, ATL-PHYS-PUB-2019-013, 2019, https://cds . cern.ch/record/2669425.

[11] ATLAS Collaboration, Standalone vertex finding in the ATLAS muon spectrometer, JINST 9 (2014) P02001, arXiv: 1311.7070 [hep-ex].

[12] ATLAS Collaboration, Search for long-lived particles produced in pp collisions at $\sqrt{s}=13 \mathrm{TeV}$ that decay into displaced hadronic jets in the ATLAS muon spectrometer, Phys. Rev. D 99 (2019) 052005, arXiv: 1811.07370 [hep-ex].

[13] ATLAS Collaboration, Search for long-lived neutral particles in pp collisions at $\sqrt{s}=13 \mathrm{TeV}$ that decay into displaced hadronic jets in the ATLAS calorimeter, Eur. Phys. J. C 79 (2019) 481, arXiv: 1902.03094 [hep-ex]. 